\title{
MMG as a Communication Channel for the Disabled
}

\author{
Sudharsan Narayanan, M. R. Mohamed Irfan, B. Geethanjali, S.Pravin Kumar \\ ${ }^{\#}$ Final Year, SSN College of Engineering \\ *Assistant Professor, SSN College of Engineering
}

\begin{abstract}
This paper describes about an improved system to provide an effective communication tool for the people who are disabled. The Mechanomyogram $(M M G)$ signal is used as a control signal for this system. Mechanomyogram signal is the superficial measurement of the low frequency vibrations that are emitted by muscles when they contract. The mentioned system acquires $M M G$ signal from biceps brachii muscle and the temporalis muscle using microphone sensor. A communication window is developed using LabVIEW software which consists of various icons or LEDs that will specify various actions which the user needs to communicate. The users can select these icons by contracting their muscle which will generate MMG that will act as a control signal and select the icons. These icons when selected will execute a respective sound file that will be played aloud using speakers, thus communicating the needs of the user.
\end{abstract}

Keywords: communication tool, mechanomyogram (MMG), disabled, microphone

\section{INTRODUCTION}

Communication among people is considered a fundamental element for surviving and preserving a good quality of life in society. Speech disabled people try to establish conversations by using symbolic languages among themselves and their caretakers. In a collective society, speech disabled people are teased by others. Also, this condition restricts the ability to express freely and quickly their urgent needs or common conversations with the rest of the society [1]. Persons with severe handicaps, especially verbal handicaps, have limited methods of adaptive control and means of communication. A variety of adaptive switches and scanning devices are available, but can have slow response times and require some tricky and trained motor coordination [2]. Several methods are developed for the communication of severely disabled, such as targeting by head movement, gaze-controlled system etc, but these systems increase the cost and complexity of the instrumentation [3]. Patients suffering from paralysis caused by advanced motor neuron disease are often unable to utter words or intelligible language. They are frequently confined to lying on their backs and are unable to help themselves; even the most basic personal needs have to be performed for them. Many such patients are highly intelligent but they are unable to express their thoughts or hold conversations due to the lack of a suitable communication channel. Although these patients may be unable to speak, there are generally a few muscles over which they have some control and whenever these muscles are exercised, for example to cause a facial twitch, jaw movement, raised eyebrow, etc, EMG signals of a few hundred microvolt vibrating at frequencies 20 - 200 $\mathrm{Hz}$ will be generated at the surface of the skin adjacent to the muscle [4].

The electromyogram (EMG) is an electrical signal observable on the surface of the muscle, which has been used very commonly to provide an alternative channel of communication for disabled people and as a control signal for prosthetic limbs [5], [6], [7], [8]. Along with the electrical signal, muscle contraction produces mechanical vibrations that may be detected at the surface of the skin. This signal is known as the mechanomyogram (MMG). The MMG is often referred to as "muscle sound", the "phonomyogram" or the "sound myogram" since it can be detected by placing a microphone over the muscle [9], [10], [11], [12].

The detected MMG is thought to be the summation of all the twitches observable at the region of detection. There are a number of advantages of using the MMG instead of the EMG for communication and control purposes. In terms of convenience and user comfort, this is preferable to single channel surface EMG recording, which typically uses three electrodes (two differential and one ground). EMG recordings also require the skin to be prepared with an alcohol swab before recordings to improve skin conductance. Since the MMG is a mechanical signal, no skin preparation is required. The signal-to-noise ratio of the MMG is typically higher than that of the EMG [13], [14]. The MMG signal acquired from biceps brachii after amplification is shown in figure 1. It is a random, noise-like signal with an approximately Gaussian amplitude distribution. The useful bandwidth of the signal is between approximately $3 \mathrm{~Hz}$ to $100 \mathrm{~Hz}$. 


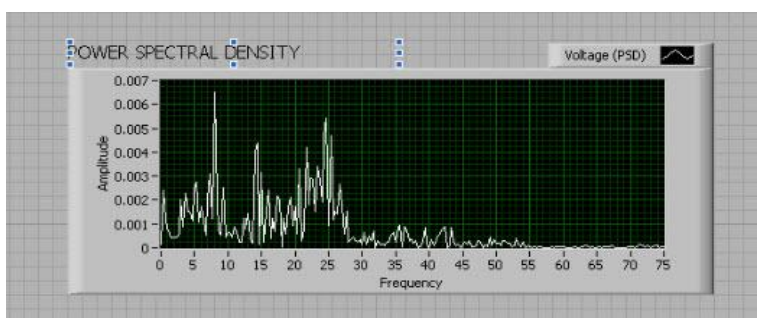

Fig. 1 Power Spectral Density (PSD) of an MMG Signal

\section{METHODOLOGY}

\section{Data Collection:}

MMG is present around an excited voluntary muscle on the surface of the skin. This enables easy selection of trigger site. The system described is used to acquire MMG from the forearm and use the mechanically generated input pulse to drive the actuators. The initial stage of the system consists of a Microphone sensor that is attached to the subject in order to receive signals from the arm and a NI USB DAQ system. A virtual LabVIEW block diagram that has a DAQ of sampling frequency $3 \mathrm{kHz}$, rectifier, smoothing filter of order 21, thresholding filter and graph Indicator with number of samples at $1 \mathrm{k}$ is generated.

MMG is acquired by a microphone-based sensor. The sensor is placed on the desired muscle and the subject is asked to contract the muscle which generates vibrations over the skin that can be picked up as MMG signals. As the muscle at the site of interest is contracted and relaxed, the diaphragm vibrates in resonance with the vibrations on the skin and carries the signal through leads. The acquired signal is shown in the figure 2 . This signal is passed through an MMG amplifier circuit. This amplifier is similar in design to a simple audio amplifier and is shown in figure 3 .

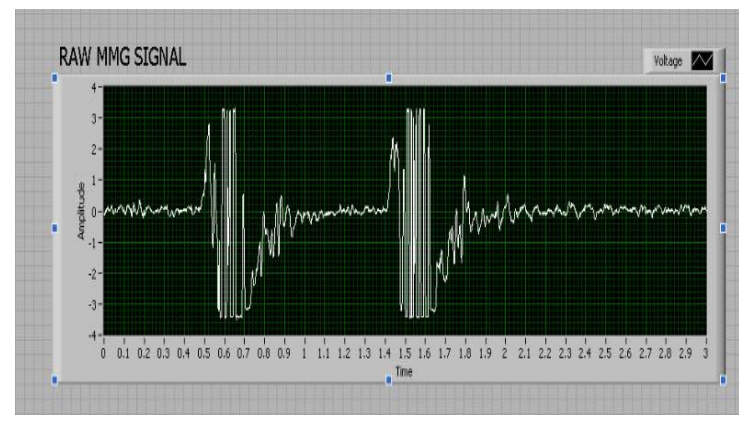

Fig. 2 MMG Signal as obtained from the circuit shown below

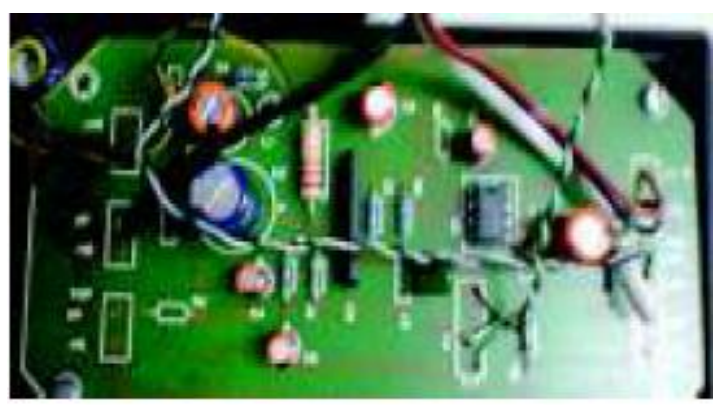

Fig. 3 Circuit used for amplifying MMG Signal from the subject

\section{Signal processing:}

The raw signal acquired is processed using virtual instrumentation software LabVIEW. In order to convert the MMG signal into a control signal that is a pulsed wave, the MMG signal is processed by a series of steps. First the signal is rectified by a squaring operator and then it is smoothened by a smoothening filter and after that using a threshold operator a pulsed wave is produced. The threshold is set accordingly dependent on the patient's ability and the type of muscle chosen for contraction. Now this pulsed wave can be used for communication process. The following figure shows the successive stages of processing. 


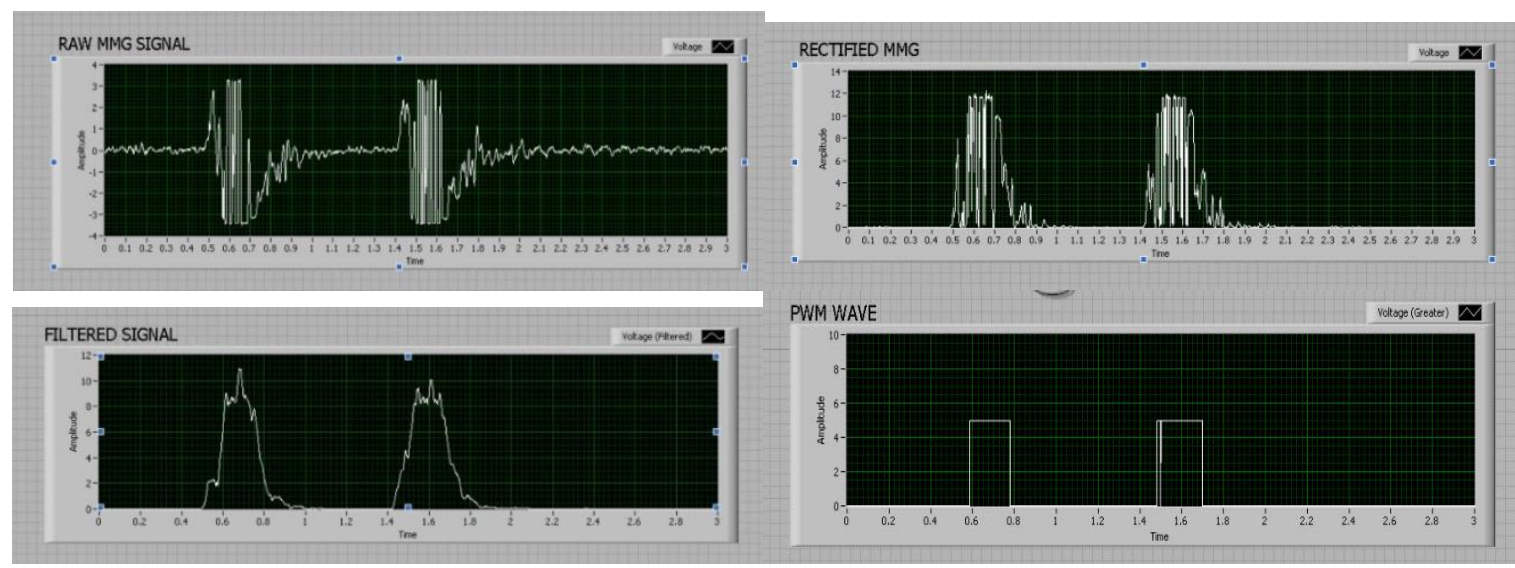

Fig. 4 LabVIEW Signal Processing of Acquired MMG Signal; includes rectification, filtering and thresholding

\section{Control logic for Communication window:}

A window is created with certain number of options or actions that a patient wishes to communicate. These icons specify the basic day today activities like I'm hungry or I'm thirsty. The patient is trained such a way that he's able to give the trigger or contract the muscle and provide the MMG signal. An array of LEDs is created. The icon or the LEDs will be switched for a particular amount of time defined by the user and then will be switched off and successive LED will glow. This action continues as long as a trigger is detected from the user. And if the patient desires to trigger a certain icon he has wait for its turn and then select it using his muscle contraction. The icon when selected will trigger a sound file corresponding to the action that the icon specifies. After an icon is selected by the user and the sound is played, the system waits for a user defined delay time and then again starts to glow the LEDs in the same order specified. Thus a patient will able to do actual communication with his muscle contraction. The LabVIEW front panel which is used as the communication window is shown in figure 5 . The block diagram of the setup is show in figure 6.

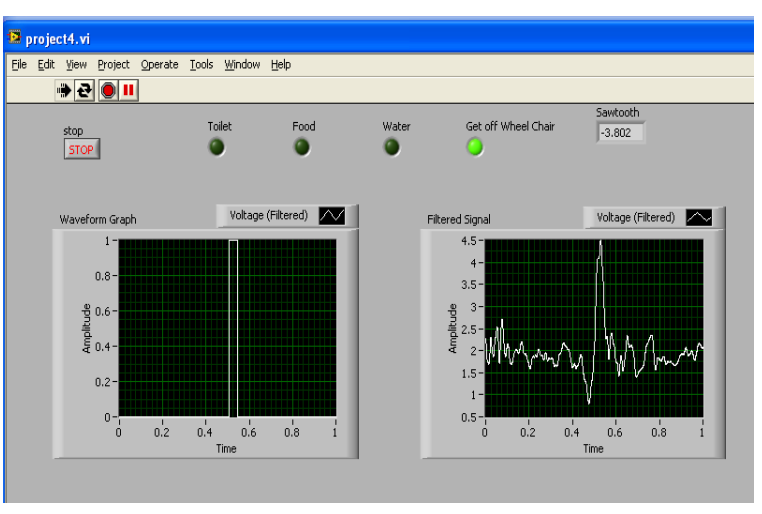

Fig. 5 LabVIEW front panel

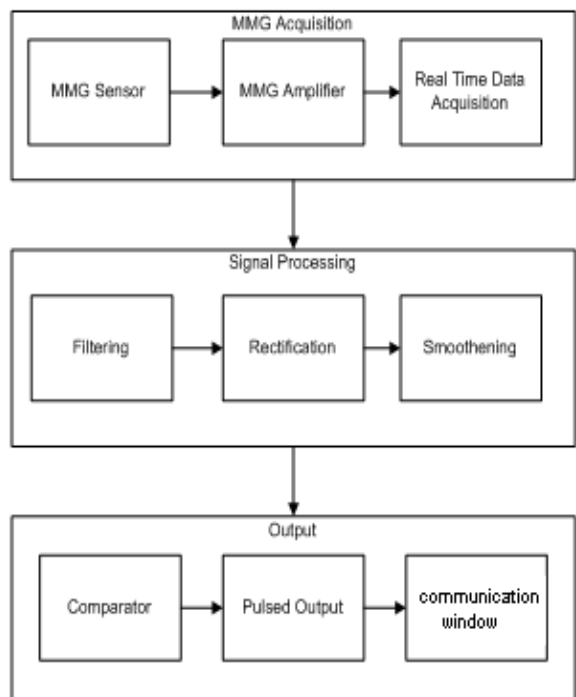

Fig. 6 Block diagram for the communication operation

\section{RESULTS}

The results of the experiment are shown in the table below. The experiment was conducted on five subjects and signals were taken from the biceps brachii muscle (A) and temporalis muscles (B). The time taken for each LED to glow is chosen to be 5 seconds which was felt comfortable by all the subjects. The number of errors for each trial was found and tabulated in table 2. 
MMG as a Communication Channel for the Disabled

TABLE 1

Parameters of the MMG acquired from two average subjects

\begin{tabular}{|c|c|c|}
\hline & Subject 1 & Subject 2 \\
\hline Amplitude & $4 \mathrm{~V}$ & $3.4 \mathrm{~V}$ \\
\hline Frequency & $10 \mathrm{~Hz}$ & $23 \mathrm{~Hz}$ \\
\hline PSD & $\begin{array}{c}\text { Concentration } \\
\text { around } 20 \mathrm{~Hz}\end{array}$ & $\begin{array}{c}\text { Concentration } \\
\text { around } 20 \mathrm{~Hz}\end{array}$ \\
\hline
\end{tabular}

TABLE 2

Errors obtained from the muscles used for each subject

\begin{tabular}{|c|c|c|}
\hline & Type of muscle & No of errors \\
\hline \multirow{2}{*}{ Subject 1 } & $\mathrm{A}$ & 1 \\
\cline { 2 - 3 } & $\mathrm{B}$ & 0 \\
\hline \multirow{2}{*}{ Subject 2 } & $\mathrm{A}$ & 0 \\
\cline { 2 - 3 } & $\mathrm{B}$ & 0 \\
\hline \multirow{2}{*}{ Subject 3 } & $\mathrm{A}$ & 0 \\
\cline { 2 - 3 } & $\mathrm{B}$ & 1 \\
\hline \multirow{2}{*}{ Subject 4 } & $\mathrm{A}$ & 1 \\
\hline \multirow{2}{*}{ Subject 5 } & $\mathrm{B}$ & 0 \\
\cline { 2 - 3 } & $\mathrm{A}$ & 2 \\
\hline
\end{tabular}

\section{DISCUSSION}

As compared to the communication systems which use alphabet boards for communication this system provides more comfortable and less frustrating method of access to the subject as they can convey their thoughts faster. Also few pulses are required from the subject for usage of the system. This system is highly suitable for those who are bed ridden and require communication of their needs to others for helping them do their day to day basic needs. The additional sound files that are installed acts as an added advantage as it can allow people to quickly and effectively understand what the subject is trying to convey. Also the use of MMG is a great advantage to the patient as the irritation caused by the frequent removal of electrodes can be eliminated. The system can be upgraded by including the alphabet board along with the icons allowing an extended range of communication.

\section{Conclusion}

The preliminary results indicate that this system can effectively help the people who cannot communicate by traditional means to communicate with others. Further improvements can be made in this system by including graphical features which will assist the users more effectively.

\section{REFERENCES}

[1] P. S. Luna et al, “Communication aid for speech disabled people using Morse codification”, EMBSBMES Conference, October 2002.

[2] John R. Lacourse and Francis C Hludik Jr, "An Eye Movement Communication-Control System for the Disabled", Transactions on Biomedical Engineering, December 1990.

[3] Hae-Jeong Park et al, “Adaptive EMG-Driven Communication for Disability”, BMESiEMBS Conference, October 1999.

[4] Jack Dinsdale and David K Chadwick, "Communication Aid for Disabled Patients makes use of Facial EMG Signals and Neural Nets", The Institution of Electrical Engineers, 1995.

[5] W. Putnam and R. B. Knapp, "Real-time computer control using pattern recognition of the electromyogram", IEEE EMBS 1993.

[6] Gwo-Ching Chang et al, "Real-time implementation of electromyogram pattern recognition as a control command of man-machine interface", October 1996

[7] S. Lee and G. Saridis, "The control of a prosthetic arm by EMG pattern recognition", IEEE Trans. Automatic Control, April 1984.

[8] R.N. Scott and P.A. Parker, "Myoelectric prostheses: state of the art", Journal of Medical Engineering \& Technology, July/August 1988.

[9] C. Orizio, "Muscle Sound: Bases for the introduction of mechanomyographic signal in muscle studies", Critical Reviews in Biomedical Engineering, 1993.

[10] M. Ouamerm et al. "Acoustic myography during voluntary isometric contraction reveals non-propagative lateral vibration", Journal of Biomechanics, 1999.

[11] M. Tarata, A. Spaepen and R. Puers, "The Accelerometer MMG Measurement Approach, in Monitoring the Muscular Fatigue", Measurement Science Review, 2001.

[12] C. Orizio et al, "Coherence Analysis Between Soundmyogram and Electromyogram”, IEEE-EMBS 1991.

[13] Yvonne Nolan1, Annraoi dePaor and Dun Laoghaire, "The Mechanomyogram as a Channel of Communication and Control for the Disabled", September 2004.

[14] N. Miyamoto and S. Oda, "Mechanomyographic and electromyographic responses of the triceps surae during maximal voluntary contractions", Journal of Electromyography and Kinesiology, 2003. 